\title{
Full “Ab-Initio" Simulation of Field Evaporation of Samples with Grain Boundaries
}

Jiayuwen $\mathrm{Qi}^{1}{ }^{1}$, Christian Oberdorfer ${ }^{1}$, Emmanuelle Marquis $^{2}$ and Wolfgang Windl ${ }^{1}$

${ }^{1}$ The Ohio State University, Columbus, Ohio, United States, ${ }^{2}$ University of Michigan, Ann Arbor, Michigan, United States

The ability to provide three-dimensional material analysis with atomic-scale insight into the chemical composition and atomic structure of materials makes atom probe tomography (APT) a unique and promising microscopy technique. Due to technological advances in instrumentation, sample preparation, and outstanding analytical capacities, APT has become a standard characterization technique on par with other high-resolution imaging techniques over the past decade.

Despite these exciting prospects, artifacts existing in the reconstruction process often limit the spatial resolution and the fidelity of atomic positioning. In order to better understand and eventually eliminate or at least minimize these artifacts, novel forward-modeling techniques of field evaporation have been developed to complement experiments and traditional projection-based reconstruction algorithms. While previous forward modeling techniques were based on a rigid lattice approximation and fixed, environment-independent evaporation field catalog, our recently introduced TAPSim-MD [1] approach combines the classic electrostatic APT modeling approach on the basis of finite elements (FE) [2] with molecular dynamic (MD) calculations in LAMMPS [3]. This combination takes into consideration key physical phenomena. Atoms in the sample tip are allowed to relax between evaporation events. A dynamic electric field allows realistic evaluation of the considerable time-dependent Coulomb forces on the tip atoms and integrates evaporation events as part of the MD simulation. An earlier version of this approach using predetermined evaporation fields to determine evaporation events and atomic relaxation through MD was already able to reproduce the experimentally observed surprising solute clustering around certain poles (e.g. for $\mathrm{Cu}$ or $\mathrm{Si}$ in $\mathrm{Al}$ ), and identify it as an artifact caused by solute surface migration from athermal relaxation [1].

Here, we take this approach to the next level by fully "ab-initio" simulating field evaporation through infield MD simulations and apply it to sample tips with grain boundaries. This is an especially interesting situation since first, it is usually speculated that bonding around grain boundaries would be in general weaker than in the bulk due to the non-ideal bonding situation, which then should lead to lower evaporation fields around the grain boundary resulting in a higher evaporation rate and focusing, both increasing the perceived intensity. Secondly, solute atoms unhappy in the bulk tend to segregate to the grain boundary, which leads to an uneven distribution of the concentration of the solute in the alloy with unknown effects on the effective local evaporation fields and their consequences on the observed detector hits. In order to examine these effects and the potential artifacts resulting from that, we have used TAPsimMD to study field evaporation of tips with grain boundaries alone or in combination with segregated solutes, whose equilibrium distribution around the grain boundary has been determined by Monte Carlo simulations. These simulations allow determining evaporation sequence and local structural effects on it from grain boundary and segregated atoms, effective evaporation field changes, and reconstruction artifacts by running the evaporation sequence through a conventional reconstruction tool. Our studied systems focus around tungsten tips with solutes such as rhenium as shown in Figure 1 [4]. 


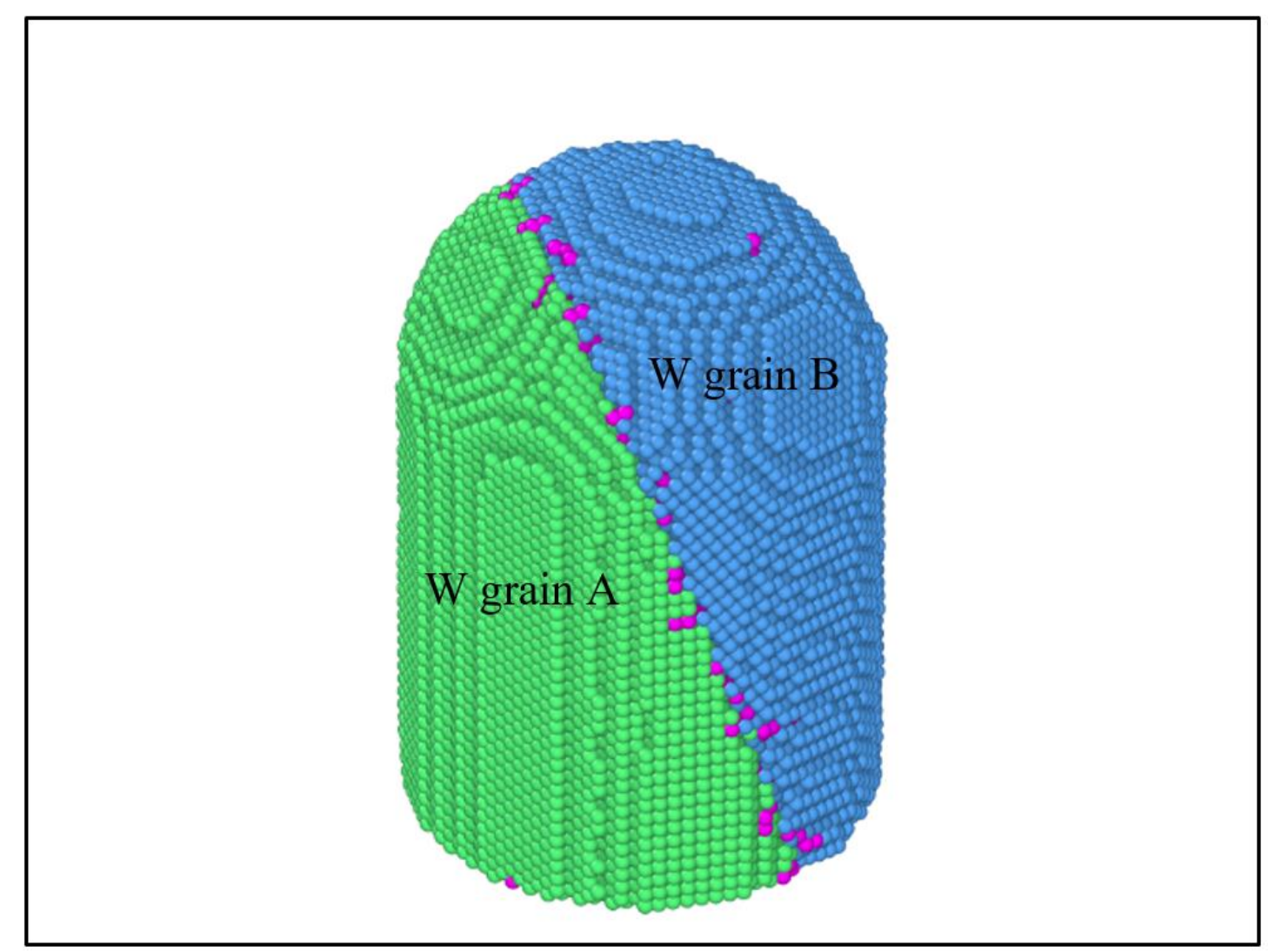

Figure 1. Simulated emitter tip with rhenium solute atoms segregated around a $\sum 5$ grain boundary in tungsten.

\section{References}

[1] C Oberdorfer et al., Mater. Charact. 146 (2018), p. 324.

[2] F Vurpillot and C Oberdorfer, Ultramicroscopy 159 (2015), p. 202.

[3] S Plimpton, J. Comput. Phys. 117 (1995), p. 19.

[4] Funding for this work was provided by Dr. Ali Sayir's portfolio within the Air Force Office of Scientific Research under grant number FA9550-19-1-0378. 